See discussions, stats, and author profiles for this publication at: https://www.researchgate.net/publication/316900537

\title{
Robust Real-Time Musculoskeletal Modeling Driven by Electromyograms
}

Article in IEEE Transactions on Biomedical Engineering · May 2017

DOI: 10.1109/TBME.2017.2704085

CITATIONS

10

3 authors:

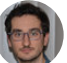

Guillaume Durandau

University of Twente

12 PUBLICATIONS 14 CITATIONS

SEE PROFILE

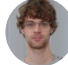

University of Twente

75 PUBLICATIONS 727 CITATIONS

SEE PROFILE

Some of the authors of this publication are also working on these related projects:

Project H2R Project View project

Project Rehabilitation Induced Neural Plasticity after Acquired Brain Injury View project
READS

245

Dario Farina

Universitätsmedizin Göttingen

717 PUBLICATIONS 18,561 CITATIONS

SEE PROFILE 


\title{
Robust Real-Time Musculoskeletal Modeling driven by Electromyograms
}

\author{
Guillaume Durandau, Student Member, IEEE, Dario Farina, Senior Member, IEEE, and Massimo \\ Sartori, Member, IEEE*
}

\begin{abstract}
Objective: Current clinical biomechanics involves lengthy data acquisition and time-consuming offline analyses and current biomechanical models cannot be used for real-time control in man-machine interfaces. We developed a method that enables online analysis of neuromusculoskeletal function in vivo in the intact human. Methods: We used electromyography (EMG)-driven musculoskeletal modeling to simulate all transformations from muscle excitation onset (EMGs) to mechanical moment production around multiple lower-limb degrees of freedom (DOFs). We developed a calibration algorithm that enables adjusting musculoskeletal model parameters specifically to an individual's anthropometry and force-generating capacity. We incorporated the modeling paradigm into a computationally efficient, generic framework that can be interfaced in real-time with any movement data collection system. Results: The framework demonstrated the ability of computing forces in $\mathbf{1 3}$ lower-limb muscle-tendon units and resulting moments about three joint DOFs simultaneously in real-time. Remarkably, it was capable of extrapolating beyond calibration conditions, i.e. predicting accurate joint moments during six unseen tasks and one unseen DOF. Conclusion: The proposed framework can dramatically reduce evaluation latency in current clinical biomechanics and open up new avenues for establishing prompt and personalized treatments, as well as for establishing natural interfaces between patients and rehabilitation systems. Significance: The integration of EMG with numerical modeling will enable simulating realistic neuromuscular strategies in conditions including muscular/orthopedic deficit, which could not be robustly simulated via pure modeling formulations. This will enable translation to clinical settings and development of healthcare technologies including real-time bio-feedback of internal mechanical forces and direct patient-machine interfacing.
\end{abstract}

Index Terms - Electromyography; Extrapolation; Joint Moment; Musculoskeletal Modeling; Real-Time.

\section{INTRODUCTION}

$S_{\text {uns }}^{\mathrm{T}}$ TUDYING the neuromusculoskeletal (NMS) mechanisms underlying human movement is a fundamental challenge. This is central to characterize movement function and how it alters with pathology, thus providing a basis for devising personalized treatments. The study of human movement typically starts from recording of experimental data including

This work was supported by the ERC Advanced Grant DEMOVE [267888]. G. Durandau and M. Sartori were with the Clinics for Trauma Surgery, Orthopedic Surgery and Plastic Surgery, University Medical Center Göttingen, DE. They now are with the Department of Biomechanical Engineering, University of Twente, NL. D. Farina is with the Department of Bioengineering, Imperial College London, UK (e-mail: g.v.durandau@utwente.nl; m.sartori@utwente.nl). whole-body kinematics, foot-ground reaction forces (GRFs) and muscle electromyograms (EMG). Computational NMS models and simulations can be subsequently established to track experimental recordings, i.e. EMGs, GRFs, and marker trajectories [1]. This enables accessing internal body variables that are not easily measured experimentally [2], e.g. muscle force [3] or joint loadings [4].

Musculoskeletal models based on inverse dynamics are currently operated offline and available in software packages such as OpenSim [5], AnyBody [6] and Biomechanics of Bodies [7]. Recent studies proposed online solutions, facilitating translation to clinical scenarios [8], [9]. In these methods, the multi-muscle recruitment problem is solved by navigating the solution space and selecting one muscle activation strategy that is optimal according to a priori defined physiological criteria, i.e. minimal sum of squared activation [10]. However, pre-defined criteria cannot encompass an individual's entire neuromuscular repertoire and its adaptations across conditions [11].

This motivated forward dynamics methods where EMG is combined with numerical simulation to account for realistic neuromuscular strategies without making assumptions on muscle recruitment. These are referred to as EMG-driven musculoskeletal models [12]-[16]. The authors and colleagues have developed and used them for estimating internal body forces [12] tightly depend on multi-muscle co-excitation, such as joint loadings [4], [17] or joint stiffness [18], [19], where inverse dynamics methods would be challenged [20], [21].

Online EMG-driven modeling has been so far proposed and tested only in restricted conditions, i.e. about one singledegree of freedom (DOF) only [22]-[25], on isometric tasks [22], and validated on the same tasks used for model calibration. Moreover, current online formulations did not model the full force-length-velocity properties of muscles [22]-[25]. This all would prevent robust translation of these solutions to real-world applications. Although a real-time twoDOF upper limb model was recently proposed [26], this was not driven by actual voluntary EMGs but operated via synthetic simulated signals. Moreover, it was tested for computational speed on a desktop computer and was not validated on the ability of blindly predicting internal joint forces.

We propose for the first time, an EMG-driven musculoskeletal modeling framework, that enables operating any musculoskeletal geometry model online and simulating the dynamics of multiple skeletal DOFs simultaneously. We tested the framework on the ability of predicting joint moments from motor tasks and DOFs that were not used for 


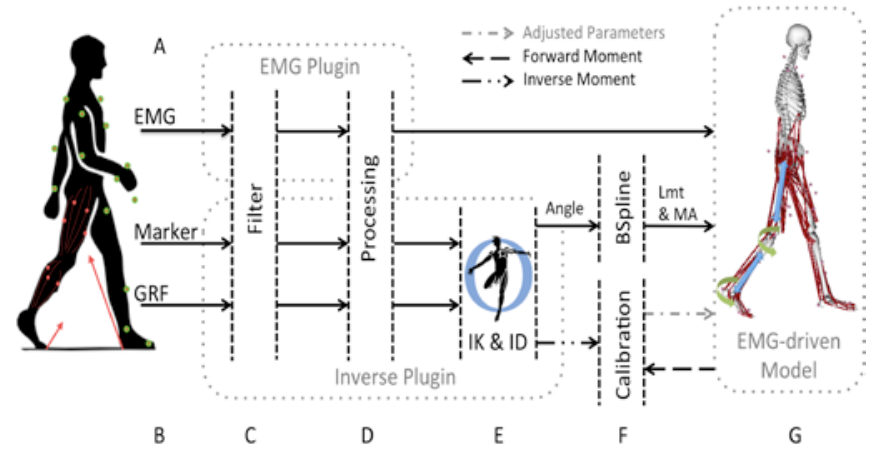

Fig. 1. Schematics of the modeling framework. It is composed of four main parts including: movement data recording (A, B), plug-in system for data processing (C-E, with real-time inverse kinematics and inverse dynamics), musculoskeletal model calibration procedure and the realtime EMG-driven musculoskeletal modeling (F, G). The Calibration procedure and the BSpline coefficients computation are performed offline. Also see Section II.

calibration, demonstrating extrapolation capacity. We also demonstrated that the framework can operate online on low power embedded systems with computational latencies that are within the physiological electromechanical delay (EMDs). Our framework realizes processing steps that are normally performed by multiple software tools while providing realtime access to internal body variables, such as muscle activation, fiber length, contraction velocity as well as musculotendon length (Lmt), moment arm (MA), force and resulting net joint DOF moments. To enable further use in the scientific community we provide open-access to movement data and simulation at simtk.org ${ }^{\dagger}$.

The paper is organized as follows: Section II presents the model structure and architecture. Section III presents the experiments conducted. Section IV-VI provide results, discussion and conclusion remarks.

\section{REAL-TIME EMG-DRIVEN MODELING}

We developed a real-time musculoskeletal modeling pipeline driven by measured EMGs and motion-capture data based on our previous work (Fig. 1) [15], [27], [28]. The pipeline first stage (see $I K \& I D$ in Fig. $1 \mathrm{E}$ ) is based on a mathematical representation of the dynamics and kinematics of the human whole-body encompassing 23 DOFs. The second stage (see BSpline in Fig. 1F), uses lower extremity joint kinematics (6 DOFs) to determine the underlying muscletendon kinematics, i.e. Lmt and MA. The third stage (see EMG-driven model in Fig. 1G), uses EMGs in conjunction with muscle-tendon kinematics to compute musculotendon force and resulting joint moments in the knee and ankle joints (Fig. 2).

The real-time framework was developed in ANSI C++ (Fig. 1). It comprises two plug-in modules for direct connection with external recording devices (Fig. 1A-B) and with the OpenSim application programming interface (API, Fig. 1E-F). Moreover, it comprises a modeling component for the computation of musculotendon kinematics based on our previously developed Multidimensional Cubic BSpline (MCBS) method (Fig. 1F) [28] as well as a component for the

\footnotetext{
${ }^{\dagger}$ https://simtk.org/projects/rems
}

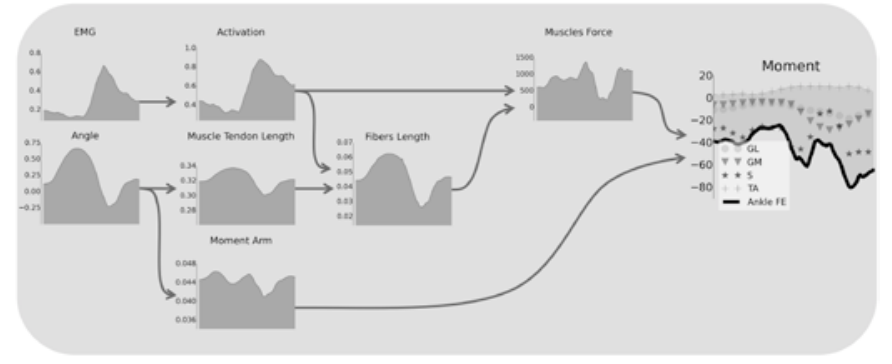

Fig. 2. Workflow of the EMG-driven musculoskeletal modeling pipeline. From EMG-excitations and joint angles to predicted internal joint moments. The diagram depicts, representatively, Soleus muscle variables and the net joint moment contributed by the muscles spanning the ankle plantar-dorsi flexion DOF. Angles are in radians, the EMGexcitations and activation are normalized. Fiber length, LMT and MA are in meter. Muscle forces are in Newton and joint moments in Newton-meter.

simulation of musculotendon dynamics based on the previously developed Calibrated EMG-informed Neuromusculoskeletal Modeling (CEINMS) method [15], [27] (Fig. 1G).

\section{A. Software Plug-In}

The first plug-in module enables TCP/IP direct connection to external EMG amplifiers (Fig. 1A). It records raw EMGs and extracts amplitude-normalized linear envelopes. The processing steps include high-pass filtering, full-wave rectification, and low-pass filtering. For each subject and muscle, the resulting EMG linear envelopes were amplitudenormalized with respect to the peak-processed values obtained from the entire set of recorded trials including both isometric maximal voluntary contractions (MVCs) and dynamic trials. This assured EMG linear envelopes always varied between 0 and 1 , an important requirement for musculotendon unit (MTU) force-production modeling. Filtered and amplitudenormalized EMGs will be referred to as muscle excitations.

The second plug-in module enables TCP/IP direct connection to external motion capture (MOCAP) systems (Fig. 1B). It records and processes three-dimensional marker trajectories and GRFs to derive joint angle and joint moment estimates via real-time inverse kinematics (IK) and inverse dynamics (ID) performed using the OpenSim API. The module low pass filters the three-dimensional marker trajectories and rotates them from the MOCAP system reference frame into the OpenSim reference frame (Fig. 1D). The OpenSim model used for the IK and ID procedure is taken from [29] and comprises 23 DOFs.

We extended the OpenSim single-thread IK algorithm into a multi-thread algorithm that produced real-time estimates (i.e., at $100 \mathrm{~Hz}$ ) of three-dimensional joint angles from filtered, rotated marker trajectories (Fig. 1E). In this, we established a direct TCP/IP connection to the MOCAP system to record markers trajectories and stream them to the OpenSim API framework (Fig 1B-E). The IK problem in OpenSim is solved via static optimization. For each time frame, three-dimensional joint angles are computed to minimize the root mean squared error (RMSE) between a set of virtual markers attached to the OpenSim musculoskeletal model anatomical landmarks and the corresponding set of experimental markers placed on the 


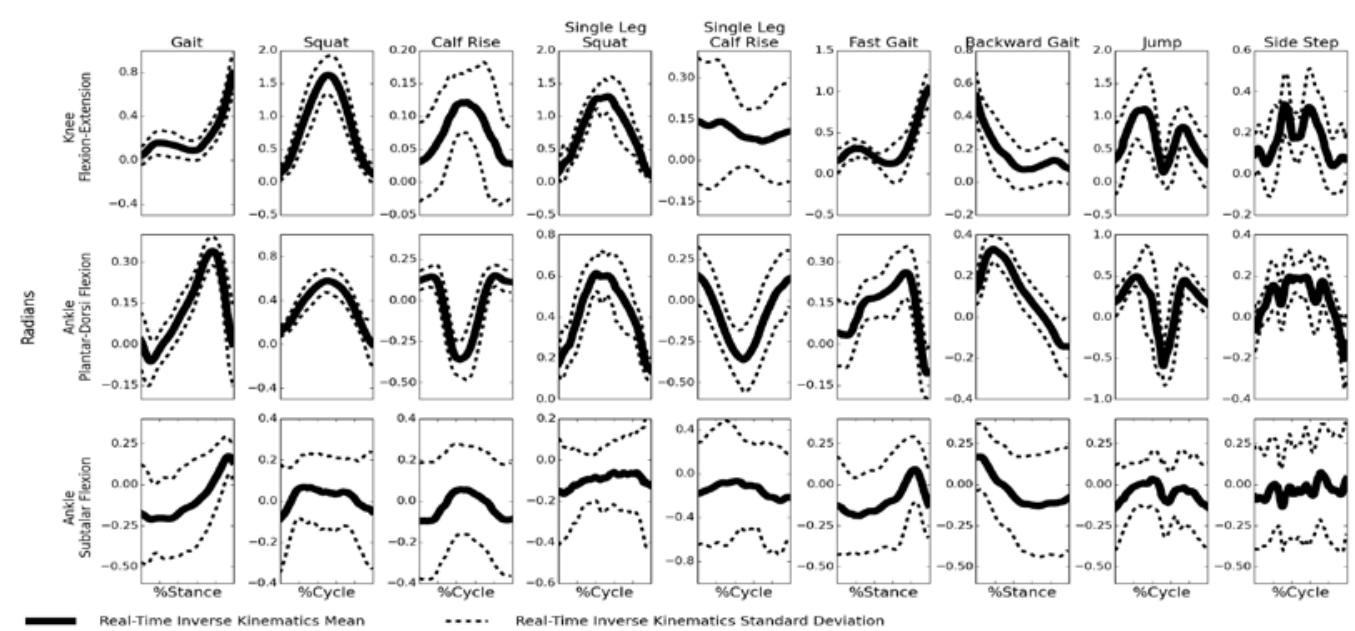

Fig. 3. Real-time inverse kinematics results. Black lines report the mean angular positions across all subjects and experiments, and the dotted line is the standard deviation. Subgraphs are organized with tasks horizontally and degrees of freedom vertically.

same anatomical landmarks of each subject [5]. To obtain real-time IK capability, we ran simultaneously multiple optimizations on different threads within a multi-stage pipeline. When a single frame of experimental marker trajectory is received, it is assigned to one thread, which performs one IK optimization. When a new experimental marker trajectory is received and the previous thread has not yet completed by the IK optimization, a new thread is established to perform concurrent optimization. The initial parameters used for the up-coming optimization stage are the latest computed DOF angles available. The plug-in also records experimental GRFs, low pass filters them and computes the resulting foot-ground center of pressure (COP, Fig. 1C). Filtered GRFs and COPs are rotated from the force plate reference frame into the OpenSim reference frame (Fig. 1D). The plug-in employs a Kalman filter [30] to process IKgenerated joint angles and computes dynamically consistent estimates of joint angular velocity and acceleration (Fig. 1D).
The Kalman filter parameters are derived as described previously [30]. Filtered and rotated GRFs, COPs as well as Kalman filtered joint angle, velocity and acceleration are streamed to the OpenSim API for the ID calculation and subsequent computation of the resulting joint moments (Fig. $1 \mathrm{E})$. We refer to these to as the "experimental moment".

\section{B. EMG-driven modeling}

The alternative pathway to joint moments is via EMG-driven musculoskeletal modeling (Fig. 1G). In this scheme, the same musculoskeletal geometry model used for the IK and ID calculations is employed (Section III). We computed EMGdependent forces for 13 MTUs spanning the knee and ankle joints. These included: semimembranosus, semitendinosus, biceps femoris long and short head, tensor fasciae latae, rectus femoris, vastus medialis, vastus intermedius, vastus lateralis, gastrocnemius medialis, gastrocnemius lateralis, soleus and tibialis anterior. We used a subset of the IK-generated whole-

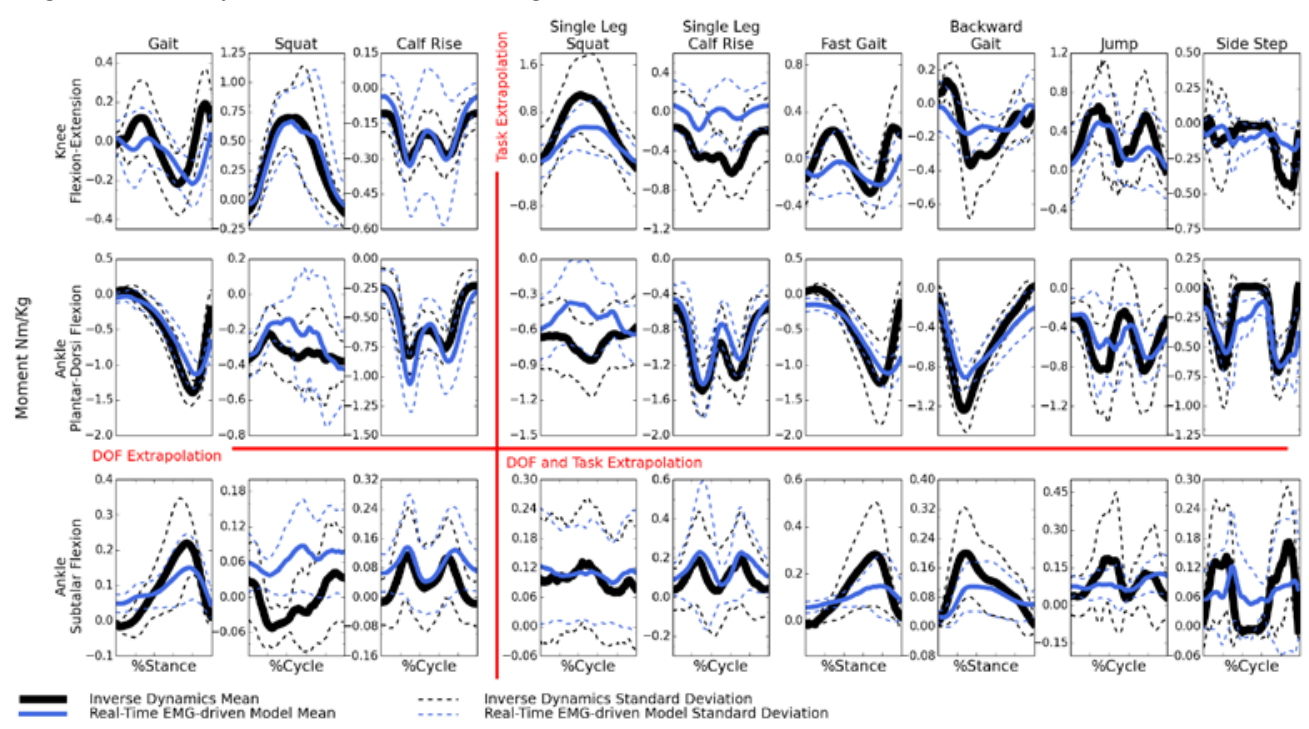

Fig. 4. Joint moments estimated via real-time EMG-driven modeling and inverse dynamics. Results report mean (solid lines) and standard deviation (dotted lines) values across all subjects and trials. Results during gait tasks are reported over the stance phase with $0 \%$ being heel strike and $100 \%$ toe-off. The remaining tasks are reported as a function of the movement cycle. The tasks to the left of the vertical red line were not used for the model calibration procedure (Section II-B), i.e. these are referred to as extrapolation tasks. The degrees of freedom (DOF) below the vertical red line were not used for calibration, i.e. extrapolated DOF. 


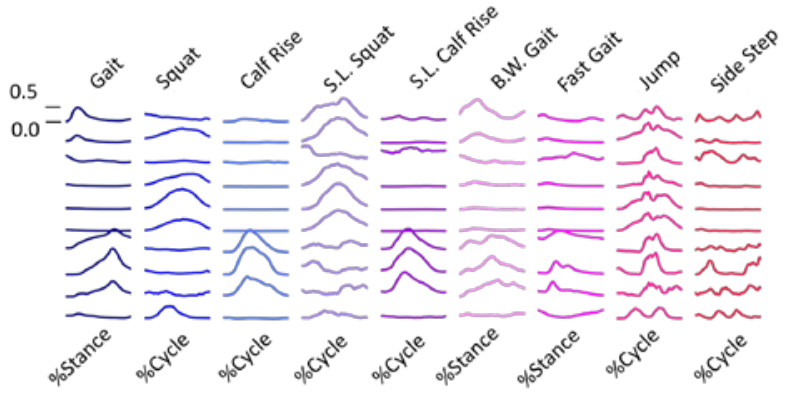

Fig. 5. Filtered and normalized EMGs across all tasks for muscles including (from top to bottom): semimembranosus, biceps femoris, tensor fasciae latae, rectus femoris, vastus medialis, vastus laterals, gastrocnemius lateralis, gastrocnemius medialis, soleus and tibia anterior.

body angle estimates. These are six lower extremity DOFs defining the kinematics of the 13 selected MTUs, including subtalar flexion, ankle flexion-extension, knee flexionextension, hip abduction, hip flexion-extension, and hip internal-external rotation [29]. IK-generated joint angles about the six selected DOFs are used to determine the underlying MTU kinematics, i.e. Lmt and MA (Fig. 1F). To achieve realtime performance we integrated in our framework the MCBS method we previously developed [28] (Fig. 1F). This synthesizes the complex MTU paths defined in large-scale OpenSim musculoskeletal geometry models into a set of MTU-specific multidimensional cubic Bsplines. This enables accurate computation of kinematic-dependent length and moment arms for all MTUs at the fastest computational speed to date, allowing the use of embedded systems with limited $\mathrm{f}$

EMG-excitation, Lmt and MA estimates are then used to compute EMG-dependent MTU force and joint moment estimates (Figs $1 \mathrm{G}$ and 2). EMG-excitations are processed via a non-linear transfer function to determine the muscle fiber twitch dynamics in response to EMG-derived muscle excitation, as previously proposed [31]. Tendons were modeled as fiber series elements of constant tendon slack length. Resulting musculotendon forces were transferred to the joint via moment arms with no modeled ligament contribution. This enabled substantial computation speed with little to no loss of accuracy with respect to elastic tendon elements in the estimation of joint moments, as we previously proved [32]. s

We developed a calibration procedure for deriving MTU parameters that determine subject specific MTU-force generating capacity and that vary nonlinearly with subject anthropometry (Fig. 1F). These included MTU-specific optimal fiber length and tendon slack length, grouped maximal muscle forces, and a global excitation-to-activation shape factor [35]. In the first stage, the calibration procedure computes BSpline coefficients necessary for the estimation of Lmt and MA. The OpenSim API is used to derive Lmt nominal values for all MTUs spanning the ankle subtalarflexion, ankle flexion-extension and knee flexion-extension DOFs. Using these data, the piecewise polynomial coefficients are computed for every order of the BSpline. The order of the BSpline depends on the number of DOFs crossed by an MTU. The second stage determines subject-specific values of optimal fiber length and tendon slack length specifically for each MTU, as previously described in [33]. An optimization procedure determines tendon slack length and optimal fiber length values so that normalized muscle fiber length and

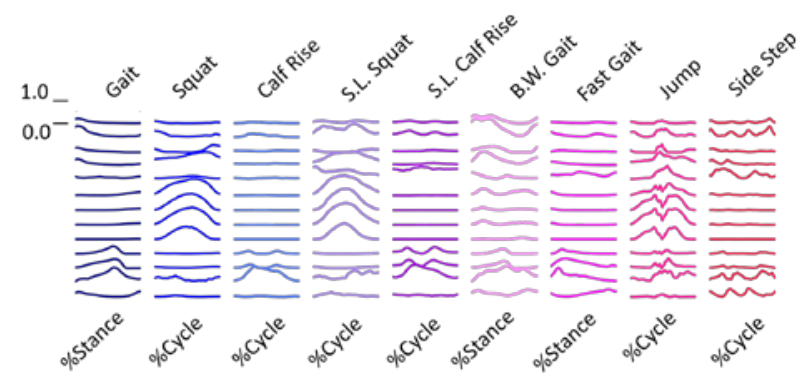

Fig. 6. Normalized musculotendon unit (MTU) force computed via the EMG-driven model across all tasks for MTUs including (from top to bottom) the semimembranosus, semitendinosus, biceps femoris long head, biceps femoris short head (BFS), tensor fasciae latae, rectus femoris, vastus medial, vastus intermedius, vastus lateralis, gastrocnemius medialis, gastrocnemius lateralis, soleus, tibia anterior.

tendon strain between the scaled and unscaled musculoskeletal geometry models are preserved across DOF functional operating ranges [33]. The third stage uses a constraint optimization to vary between pre-defined boundaries the EMG-to-activation shape factor parameter (i.e. between -3 and 0 ), the MTU maximal isometric force (i.e. scaled by factors between 0.5 and 1.5) and further refine the previous estimates of optimal fiber length (i.e. within $\pm 2.5 \%$ of its initial value) and tendon slack length (i.e. within $\pm 5 \%$ of its initial value). Parameters are varied using a simulated annealing procedure [34] until the discrepancy between experimental and predicted joint moments is minimized over a range of calibration trials (Section III). We developed a graphical user interface (GUI) to enable real-time visual feedback of modeling steps including IK and ID calculations, EMG-muscle excitation processing as well as EMG-driven model-based estimation of MTU and joint variables. The video available in the supplementary material shows the real-time modeling framework being used on one individual subject.

\section{EXPERIMENTAL PROCEDURES}

The University Medical Center Göttingen Ethical Committee approved all experimental procedures. Five healthy men (see Table I) volunteered for this investigation after providing signed informed consent. Data were recorded and processed in real-time using the modeling framework described in Section II, depicted in Fig. 1, and displayed in supplementary video.

EMGs were recorded using a 256-channel EMG amplifier (OTBioelettronica, Italy) at $2048 \mathrm{~Hz}$. The high-pass filter was a second-order Butterworth filter with $30 \mathrm{~Hz}$ cut-off. The lowpass filter was a second-order Butterworth with a $4 \mathrm{~Hz}$ cut-off. We recorded EMG signals from 10 muscle groups including: rectus femoris, lateral and medial hamstrings, vastus medialis and lateralis, tensor fasciae latae, gastrocnemious medialis and lateralis, soleus and tibialis anterior. Muscle group EMGs were allocated to individual MTUs defined in the modeling framework (Section II.B). In this allocation, two MTUs that shared the same innervation and contributed to the same mechanical action were assumed to have the same EMG pattern. According to this convention the lateral hamstring EMGs drove both the biceps femoris short head and long head MTUs. The medial hamstring EMGs drove both the semimembranosus and the semitendinosus MTUs. The vastus intermedius EMG activity was derived as the mean between 
the vastus lateralis and vastus medialis EMGs [15]. All remaining MTUs had dedicated EMG channels. A set of 29 retroreflectives markers was placed on the trunk and lower extremity, as previously described [15]. Three-dimensional marker trajectories were recorded using a seven-camera motion capture system (Qualisys, Göteborg, Sweden) at $128 \mathrm{~Hz}$. Foot-ground reaction forces (GRFs) were recorded using two in-ground force plates (Bertec, Columbus, USA) at 2048Hz. The low-pass filter used for both marker and GRF data was a second order Butterworth filter with $10 \mathrm{~Hz}$ cut-off and a time group delay of $0.1-25 \mathrm{~ms}$ with average delays in the order of $20 \mathrm{~ms}$.

The subjects performed a static standing trial. The recorded marker trajectories were used to scale an OpenSim generic musculoskeletal model to match each individual subject's anthropometry. MVC trials consisting of isometric contractions were performed for each muscle group for EMG normalization. The subjects performed three model calibration trials including one static standing trial, one single repetition of forward gait at self-selected speed, and one single repetition of knee squat followed by calf rise. The model calibration procedure (Section II) was performed to minimize discrepancy between predicted and experimental moments about the knee flexion-extension and ankle plantar-dorsi flexion DOFs. The subtalar-flexion DOF was not included in the calibration procedure. Validation trials included five additional repetitions of the calibration tasks (excluding the static standing task) as well as five repetitions of novel motor tasks including: backward gait at self-selected speed, fast forward gait, knee squat, single-leg knee squat, calf rise, single-leg calf rise, knee squat followed by vertical jump, and side stepping. Motor tasks were chosen to underlie a variety of different neuromuscular strategies and produce a range of dynamic joint moments across knee and ankle joint DOFs.

The whole real-time modeling framework (i.e. EMGprocessing, IK, ID, and EMG-driven modeling, Fig. 1) was operated on a laptop with dual-core processing unit $(2.60 \mathrm{GHz})$ and 16GB of RAM memory. Tests were also repeated using an embedded system (Raspberry Pi 2, Raspberry Pi Foundation, UK), which is a single board computer with a four-core processing unit $(900 \mathrm{MHz})$ and $1 \mathrm{~GB}$ of RAM memory. In this, joint angles and EMGs were read from file, i.e. we did not employ real-time EMG processing and IK computation. Three tests were performed for validating the framework capabilities.

\section{RESULTS}

The first test verified the framework ability of computing joint angular positions in real-time via IK. Angles estimates about 23 articular joint DOFs were produced at an average rate of $168 \pm 141 \mathrm{~Hz}$. Fig. 3 reports values derived about the knee flexion-extension, ankle plantar-dorsi flexion, and ankle subtalar flexion across all motor tasks. These are the DOFs employed in the subsequent EMG-driven modeling pipeline. IK-generated angles reflect literature values across forward gait [35], backward gait [3], and squat tasks [36]. Table I summarizes locomotion speeds performed by all subjects as well as each individual's anthropometry properties. The Table I also show how self-selected locomotion speeds largely varied across participants generating a variety of different motor conditions to be predicted by the framework.

The second test (Fig. 4) verified the real-time framework ability of estimating joint moments in real-time using the EMG-driven modeling pipeline using experimental EMGexcitations (Fig. 2) and IK-angles (Fig. 3). Results showed estimated joint moments being in agreement with ID generated joint moments (reference) derived using experimental GRFs and IK angles. Fig. 4 shows the model ability of predicting moments during novel repetitions of the calibration trials including gait at self-selected speed, knee squat with subsequent calf rise. Moreover, Fig. 4 also shows the model ability of extrapolating beyond calibration conditions. That is, to completely unseen motor tasks (i.e. extrapolation capacity: backward gait, side step, ingle leg squat with calf rise, fast gait and vertical jump), and about one unseen DOF (i.e. ankle subtalar flexion). The largest Pearson

TABLE I

PARTICIPANTS' ANTHROPOMETRIC PROPERTIES AND LOCOMOTION SPEEDS

\begin{tabular}{ccccccc}
\hline \multirow{2}{*}{ Participant } & \multirow{2}{*}{$\begin{array}{c}\text { Age } \\
\text { (years })\end{array}$} & \multirow{2}{*}{$\begin{array}{c}\text { Height } \\
(\mathrm{m})\end{array}$} & \multirow{2}{*}{$\begin{array}{c}\text { Weight } \\
(\mathrm{Kg})\end{array}$} & \multicolumn{3}{c}{ Gait Speed $(\mathrm{m} / \mathrm{s})$} \\
\cline { 5 - 7 } & 26 & 1.77 & 73 & 0.66 & 0.74 & 0.59 \\
\hline 1 & 31 & 1.82 & 70 & 0.68 & 0.93 & 0.43 \\
2 & 34 & 1.82 & 67 & 0.65 & 0.70 & 0.31 \\
3 & 29 & 1.71 & 73 & 0.58 & 0.84 & 0.49 \\
4 & 28 & 1.86 & 85 & 0.66 & 0.96 & 0.63 \\
\hline 5 & &
\end{tabular}

coefficients $r=0.9 \pm 0.07$ was observed at the ankle plantardorsi flexion DOF during gait at self-selected speed. The smallest root mean square error (RMSE) was observed at the subtalar flexion DOF $(0.01 \pm 0.01 \mathrm{Nm} / \mathrm{kg})$ during the single leg squat task. Pearson coefficients were always greater than $\mathrm{r}=$ $0.43 \pm 0.36$ with least favorable values observed at the knee flexion-extension DOF during gait at self-selected speed. The RMSE was always smaller than $0.37 \pm 0.12 \mathrm{Nm} / \mathrm{kg}$ with least favorable values observed at the knee flexion-extension DOF during the single leg calf rise task. The EMG-driven model prediction accuracy during the unseen motor tasks was comparable to that observed during novel trials of the same type used for calibration. The RMSE and $r$ variation from calibration to extrapolation trials was $0.02 \mathrm{Nm} / \mathrm{kg}$ and 0.07 respectively at the knee flexion-extension, $0.003 \mathrm{Nm} / \mathrm{kg}$ and 0.06 at the ankle plantar-dorsi flexion, and $0.25 \mathrm{Nm} / \mathrm{kg}$ and 0.12 at the ankle subtalar flexion. The task that displayed largest prediction accuracy variation between calibration and extrapolation tasks was the single leg knee squat with calf rise. The joint moments predicted both using EMG-driven modeling and ID well reflected normative values found in literature for tasks including gait [37], backward gait [38] and squat [39].

Fig. 5 shows the EMG-excitations used for joint moment prediction across all tasks and muscles and reported for one subject. Excitations were found to assume values comparable for the forward gait[40], backward gait [41] and squat [39], for which literature data are available. During the knee squat, excitations from the quadriceps group assumed substantially high values in the knee extension part of the task. Similarly, calf muscle excitations assumed larger values during the calf 
raising part of the tasks. The jump task had comparable excitation patterns to the squat task particularly at the beginning and the end of the task.

Fig. 6 shows the normalized force predicted for all MTUs across all motor tasks and reported for one subject. Results showed values matching literature data for gait [42] and squat tasks [39] for which values for comparison are available. Importantly, Fig. 5 and 6 highlight the non-proportionality existing between EMG-excitations and resulting forces, where modulations in EMG-excitations does not always correspond to a linear modulation at the force level. This reflects the nonlinear EMG-to-activation transfer function (Section II) and the Hill-type viscoelasticity via force-length-velocity relationship.

The third test (Figs 7 and 8) quantified the framework realtime computation performance when operated both on a laboratory desktop computer and on an embedded system. We used metrics including: the mean computation time and standard deviation measured across all simulation frames from all subjects and tasks, the maximal expected computation time within a 95\% confidence interval assuming computation time frames with a normal Gaussian distribution, and the maximal expected computation time with a 90\% confidence interval with no assumption on the computation time frame distribution, i.e. using the Chebyshev's Theorem. Fig. 7 shows the computation time of the different components of the realtime EMG-driven pipeline (Fig. 1) on a desktop computer.

The MTU kinematics component (Fig. 1F) executed with a mean computation time of $0.4 \pm 0.47 \mathrm{~ms}$ with $95 \%$ of the samples being computed within $1.5 \mathrm{~ms}$. The inverse kinematics component (Fig. 1E) executed with a computational time of $10.1 \pm 8.5 \mathrm{~ms}$ with $95 \%$ of the samples being computed within 28ms. The EMG-driven model (Fig. 1G) executed in $0.301 \pm 0.65 \mathrm{~ms}$ with $95 \%$ of the samples being produced within 1.6ms. Fig. 7 also shows the total delay from the EMG recording time to the multi-DOF moment computation, with mean delay being $35 \pm 11 \mathrm{~ms}$ and with $95 \%$ of the samples being produced within $55 \mathrm{~ms}$.

Fig. 8 shows the computational time of the EMG-driven model and the MTU spline on the Raspberry Pi 2 embedded system. The MTU kinematics component (Fig. 1F) operated in

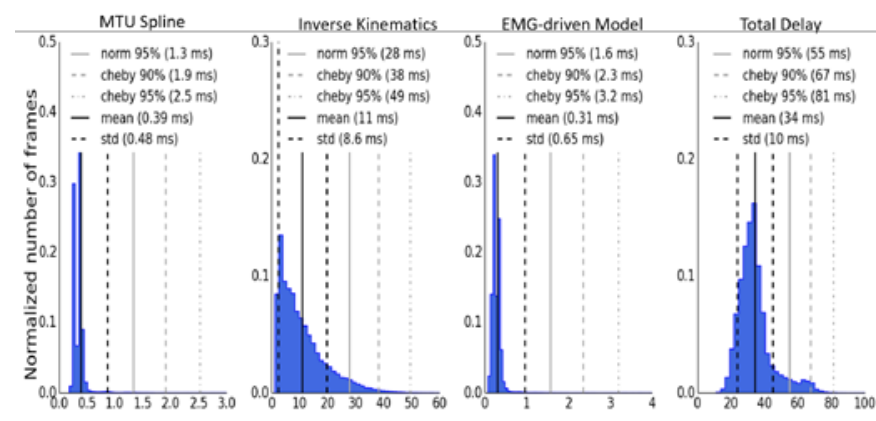

Computation Time (ms)

Fig. 7. Computation time on a desktop computer. The histograms (starting from left) respectively depict computation times for the MTU spline, inverse kinematics, and EMG-driven model component as well as the total delay between EMG sampling time and multi-DOF moment computation. The inverse dynamics computation time is not reported as this is constant and does not add substantial latency to the workflow.

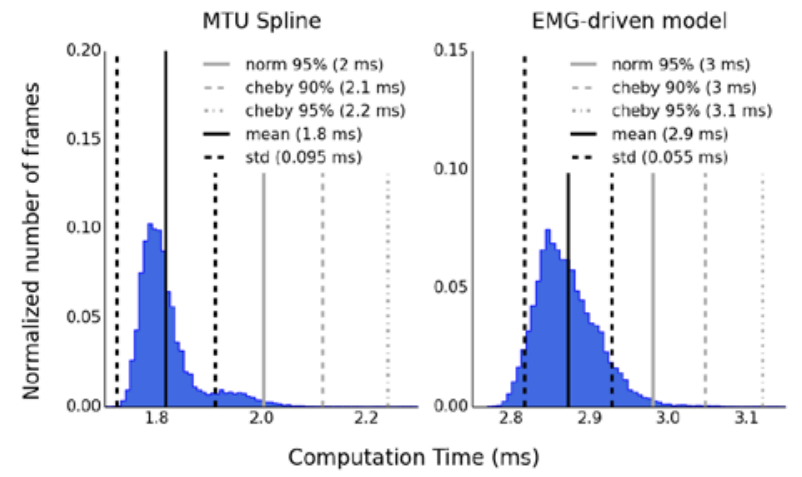

Fig. 8. Computation time on the Raspberry Pi 2. The left-hand histogram depicts the computation time of MTU spline component. The right-hand histogram depicts the computation time of the EMG-driven model component.

$4.3 \pm 0.2 \mathrm{~ms}$ with $95 \%$ of the samples being produced within 4.7ms. The EMG-driven model (Fig. 1G) operated in $2.7 \pm 0.48 \mathrm{~ms}$ with $95 \%$ of the samples being produced within $3.6 \mathrm{~ms}$. The video in the supplementary material displays the framework data recording, processing and musculoskeletal simulation capacity in real-time.

\section{DISCUSSION}

We developed and validated a real-time framework for modeling and simulating the dynamics of the human NMS system using EMG-driven modeling. The real-time framework enables recording and processing movement data (marker trajectories, GRF, EMGs) and determining reference threedimensional joint angles and moments via real-time IK and ID. Moreover, it enables simulating how EMG-controlled muscle contractions transfer mechanical force to skeletal structures instantly during an individual's movement. In this, EMGs enable simulating realistic subject-specific neuromuscular strategies across different individuals in conditions also including muscular/orthopedic deficit, which could not be robustly simulated via pure modeling formulations [43]. In this study, we calibrated and tested the EMG-driven modeling pipeline using a lower extremity musculoskeletal geometry model with six DOFs (Section II). However, the proposed framework enables real-time simulation of any musculoskeletal geometry model generated using the OpenSim modeling software package. ${ }^{\S}$

The proposed framework enabled for the first time, robust estimation of muscle-contributed joint moments about multiple DOFs simultaneously, during unseen dynamic motor tasks and DOF as well as using low power portable embedded systems. The joint moment estimation ability over the unseen motor tasks and DOFs was comparable to that observed during novel repetition of the calibration tasks and DOFs (Section II). These results support the possibility of translating the proposed EMG-driven musculoskeletal modeling technique to real-world applications, i.e. computeraided motor diagnosis and rehabilitation, human-machine interfacing, model-based control of assistive devices.

\footnotetext{
${ }^{\S}$ http://simtk-confluence.stanford.edu:8080/display/OpenSim/ Musculoskeletal+Models
} 
Results from the first test (Fig. 3) revealed large variability about the subtalar angular position highlighting this DOF predominant function of leg stabilization. This appears to be an important element especially when comparing single legsquat with respect to double leg-squat tasks (Fig. 3). These tasks display substantially different ankle moment trends (i.e. see ID moments in Fig. 4). This is explained by single legsquat tasks requiring larger knee joint moment production (see knee extensors function, Figs 5-6) and greater ankle stabilization function (see ankle antagonist muscle coactivation, Fig. 5). In this, estimation accuracy of fine ankle moment modulation was limited in our modeling framework by the fact that not all ankle muscles were recorded. However, despite current limits, the proposed modeling formulation was able to provide joint moment estimates in close agreement to reference data (Fig. 4). The results also revealed that the EMG-driven model better predicted the ankle plantar-dorsi flexion moments than the knee flexion-extension moments. This is due to the larger number of muscle prime movers in the knee than in the ankle. It is important to stress that the central requirement in our system was to achieve real-time performance, for which we needed to address computational challenges across all modeling and processing stages (i.e. Figs 1-2). These included on-line data acquisition and filtering introducing data losses and phase shifts respectively. In this context, we employed standard TCP/IP data acquisition protocols not specifically designed for hard real-time performances. This all limited the prediction accuracy of our system when compared to previous offline studies [12], [14], [15]. Future work will develop ad-hoc data acquisition and processing hardware and software systems, which will better enable handling real-time constraints.

Future work will investigate the real-time modeling formulation with a larger set of recorded EMG channels spanning the knee and quantify the associated model prediction sensitivity. Also, future work will explore whether high-density EMG can enable better estimates of muscle activity especially important during tasks underlying finecontrol of muscle excitation and small modulations of force.

The ability of predicting muscle forces while distributing their force output along all spanned DOFs simultaneously enables addressing the indeterminacy of the muscle force distribution problem, which has been achieved here for the first time in real-time. We previously showed that EMGdriven models calibrated with respect to different single-DOFs generated different MTU force solutions for the same input data and MTU set [15]. On the other hand, our proposed realtime multi-DOF modeling formulation provides a unique MTU force solution that satisfies all DOFs simultaneously and is therefore more generalizable across novel conditions. This was reflected by the model ability of extrapolating both taskwise and DOF-wise (Fig. 4).

The ability of operating the modeling pipeline in real-time is important in the context of human-machine interfacing for wearable assistive devices as it would enable predicting internal body forces and the intended movement before they actually manifest in the human body. This would enable supporting individuals with reduced motor abilities but with detectable electrophysiological activity. Conversely, systems that operate on the basis of the detection of externally measurable forces (i.e., limb orthosis interaction force) would not provide support until the user has produced detectable interaction force.

Results also revealed that the elements contributing to the total computational time included: (1) the motion capture system and IK algorithm, which relies on static optimization and therefore required substantial computational power and (2) the EMG recording system we used, which sends 30mspackets of data over TCP/IP. The EMG-driven modeling pipeline used only a small portion of the total computation time, as depicted in Figs 7-8. Future work will use fully integrated EMG and position sensors, to decrease the computational latency and assure enhanced real-time capability using wearable solutions.

The computational performance obtained using the embedded system (Fig. 8) revealed that $90 \%$ of predicted frames were computed with latencies comparable to those derived using a laptop system. Peak latencies were observed to be one order of magnitude larger than using a laptop computer system, however only in a small percentage of the cases.

Future work will investigate the possibility of predicting multiple mechanical variables including those tightly dependent on muscle co-excitation such as joint stiffness [44]. This will underlie the employment of series elastic tendon elements as previously described [44] and ligaments, thus enabling the accurate estimation of a greater range of mechanical variables. Future work will also focus on the use of co-excitation primitives [16] for relaxing sensoryconstraints, i.e. the need for recording from large sets of muscles. Applications will also include the interfacing of our proposed framework with wearable assistive technologies for restoring (robotic exoskeletons) or replacing (artificial limbs) [43] lost motor capacity. In this context, previously proposed offline NMS modeling formulations demonstrated to successfully capture patient-specific musculoskeletal function in conditions including cerebral palsy [45][46],stroke [14] or quadriceps weakness [43]. In this, important muscle functional abnormalities could be modeled by optimizing the passive muscle stiffness parameters (i.e. muscle contractures) or using velocity-dependent feedback controllers (i.e. spasticity) [45].

\section{CONCLUSION}

We proposed and extensively validated an online EMG-driven musculoskeletal modeling framework that simulates the dynamics of multiple muscular and skeletal DOFs concurrently. This will enable (1) filling the gap between data collection and advanced analysis, (2) out-of-the-lab analysis for understanding human movement beyond constrained laboratory conditions, and (3) translation to the clinics and to assistive technologies.

\section{REFERENCES}

[1] M. G. Pandy, "Computer modeling and simulation of human movement," Annu. Rev. Biomed. Eng., vol. 3, no. 1, pp. 245-273, 2001.

[2] M. G. Hoy, F. E. Zajac, and M. E. Gordon, “A musculoskeletal model of the human lower extremity: the effect of muscle, tendon, and moment arm on the moment-angle relationship of musculotendon actuators at the hip, knee, and ankle,” J. Biomech., vol. 23, no. 2, pp. 157-169, 1990.

[3] S. S. M. Lee, A. S. Arnold, M. de Boef Miara, A. A. Biewener, and J. M. Wakeling, "Accuracy of gastrocnemius muscles forces in walking and running goats predicted by one-element and two-element Hill-type models,” J. Biomech., vol. 46, no. 13, pp. 2288-2295, 2013. 
[4] P. Gerus et al., "Subject-specific knee joint geometry improves predictions of medial tibiofemoral contact forces," J. Biomech., vol. 46, no. 16, pp. 2778-2786, 2013.

[5] S. L. Delp et al., "OpenSim: open-source software to create and analyze dynamic simulations of movement," Biomed. Eng. IEEE Trans., vol. 54, no. 11, pp. 1940-1950, 2007.

[6] M. Damsgaard, J. Rasmussen, S. T. Christensen, E. Surma, and M. De Zee, "Analysis of musculoskeletal systems in the AnyBody Modeling System,” Simul. Model. Pract. Theory, vol. 14, no. 8, pp. 1100-1111, 2006.

[7] Prosim, "Biomechanics of Bodies.” 2015.

[8] A. J. den Bogert, T. Geijtenbeek, O. Even-Zohar, F. Steenbrink, and E. C. Hardin, "A real-time system for biomechanical analysis of human movement and muscle function,” Med. Biol. Eng. Comput., vol. 51, no. 10, pp. 1069-1077, 2013.

[9] A. Murai, K. Kurosaki, K. Yamane, and Y. Nakamura, "Musculoskeletalsee-through mirror: Computational modeling and algorithm for wholebody muscle activity visualization in real time," Prog. Biophys. Mol. Biol., vol. 103, no. 2, pp. 310-317, 2010.

[10]R. D. Crowninshield and R. A. Brand, "A physiologically based criterion of muscle force prediction in locomotion,” J. Biomech., vol. 14, no. 11, pp. 793-801, 1981.

[11]W. Herzog and T. R. Leonard, "Validation of optimization models that estimate the forces exerted by synergistic muscles," J. Biomech., vol. 24, pp. 31-39, 1991.

[12]D. G. Lloyd and T. F. Besier, "An EMG-driven musculoskeletal model to estimate muscle forces and knee joint moments in vivo," J. Biomech., vol. 36, no. 6, pp. 765-776, 2003.

[13]L. L. Menegaldo, L. F. de Oliveira, and K. K. Minato, "EMGD-FE: an open source graphical user interface for estimating isometric muscle forces in the lower limb using an EMG-driven model," Biomed. Eng. Online, vol. 13, no. 1, p. 1, 2014.

[14]Q. Shao, D. N. Bassett, K. Manal, and T. S. Buchanan, "An EMG-driven model to estimate muscle forces and joint moments in stroke patients," Comput. Biol. Med., vol. 39, no. 12, pp. 1083-1088, 2009.

[15] M. Sartori, M. Reggiani, D. Farina, and D. G. Lloyd, "EMG-Driven Forward-Dynamic Estimation of Muscle Force and Joint Moment about Multiple Degrees of Freedom in the Human Lower Extremity,” vol. 7, no. 12, 2012.

[16] M. Sartori, L. Gizzi, D. G. Lloyd, and D. Farina, “A musculoskeletal model of human locomotion driven by a low dimensional set of impulsive excitation primitives," Front. Comput. Neurosci., vol. 7, no. June, pp. 122, 2013.

[17] A. L. Kinney, T. F. Besier, D. D. D’Lima, and B. J. Fregly, “Update on grand challenge competition to predict in vivo knee loads," J. Biomech. Eng., vol. 135, no. 2, p. 21012, 2013.

[18] M. Sartori, M. Maculan, C. Pizzolato, M. Reggiani, and D. Farina, "Modeling and simulating the neuromuscular mechanisms regulating ankle and knee joint stiffness during human locomotion," $J$. Neurophysiol., vol. 114, no. 4, pp. 2509-2527, 2015.

[19]E. J. Perreault, R. F. Kirsch, and P. E. Crago, "Voluntary control of static endpoint stiffness during force regulation tasks,” J. Neurophysiol., vol. 87, no. 6, pp. 2808-2816, 2002.

[20]S. Heintz and E. M. Gutierrez-Farewik, "Static optimization of muscle forces during gait in comparison to EMG-to-force processing approach," Gait Posture, vol. 26, no. 2, pp. 279-288, 2007.

[21]J. P. Walter et al., "Muscle synergies may improve optimization prediction of knee contact forces during walking," J. Biomech. Eng., vol. 136, no. 2, p. 21031, 2014.

[22] K. Manal, R. V Gonzalez, D. G. Lloyd, and T. S. Buchanan, "A real-time EMG-driven virtual arm,” Comput. Biol. Med., vol. 32, no. 1, pp. 25-36, 2002.

[23] K. Manal, K. Gravare-Silbernagel, and T. S. Buchanan, "A real-time EMG-driven musculoskeletal model of the ankle,” Multibody Syst. Dyn., vol. 28, no. 1-2, pp. 169-180, 2012.

[24]E. E. Cavallaro, J. Rosen, J. C. Perry, and S. Burns, "Real-time myoprocessors for a neural controlled powered exoskeleton arm.," IEEE Trans. Biomed. Eng., vol. 53, no. 11, pp. 2387-2396, 2006.

[25]C. Fleischer and G. Hommel, "A human-exoskeleton interface utilizing electromyography,” IEEE Trans. Robot., vol. 24, no. 4, pp. 872-882, 2008.
[26]E. K. Chadwick, D. Blana, A. J. van den Bogert, R. F. Kirsch, and others, "A real-time, 3-D musculoskeletal model for dynamic simulation of arm movements," IEEE Trans. Biomed. Eng., vol. 56, no. 4, pp. 941-948, 2009.

[27]C. Pizzolato et al., "CEINMS: A toolbox to investigate the influence of different neural control solutions on the prediction of muscle excitation and joint moments during dynamic motor tasks," J. Biomech., vol. 48, no. 14, pp. 3929-3936, 2015.

[28] M. Sartori, M. Reggiani, A. J. van den Bogert, and D. G. Lloyd, "Estimation of musculotendon kinematics in large musculoskeletal models using multidimensional B-splines,” J. Biomech., vol. 45, no. 3, pp. 595-601, 2012.

[29] S. L. Delp, J. P. Loan, M. G. Hoy, F. E. Zajac, E. L. Topp, and J. M. Rosen, "An interactive graphics-based model of the lower extremity to study orthopaedic surgical procedures," IEEE Trans. Biomed. Eng., vol. 37, no. 8, pp. 757-767, 1990.

[30]F. De Groote, T. De Laet, I. Jonkers, and J. De Schutter, "Kalman smoothing improves the estimation of joint kinematics and kinetics in marker-based human gait analysis,” vol. 41, pp. 3390-3398, 2008.

[31] K. Manal and T. S. Buchanan, "A one-parameter neural activation to muscle activation model: estimating isometric joint moments from electromyograms," J. Biomech., vol. 36, no. 8, pp. 1197-1202, 2003.

[32] M. Sartori, M. Reggiani, E. Pagello, and D. G. Lloyd, "Modeling the human knee for assistive technologies," IEEE Trans. Biomed. Eng., vol. 59, no. 9, pp. 2642-2649, 2012.

[33] C. R. Winby, D. G. Lloyd, and T. B. Kirk, "Evaluation of different analytical methods for subject-specific scaling of musculotendon parameters,” J. Biomech., vol. 41, no. 8, pp. 1682-1688, 2008.

[34]W. L. Goffe, G. D. Ferrier, and J. Rogers, "Global optimization of statistical functions with simulated annealing,” J. Econom., vol. 60, no. 1-2, pp. 65-99, 1994.

[35]R. Grasso, L. Bianchi, and F. Lacquaniti, "Motor patterns for human gait: backward versus forward locomotion,” J. Neurophysiol., vol. 80, no. 4, pp. 1868-1885, 1998.

[36] S. A. Horan, S. L. Watson, C. P. Carty, M. Sartori, and B. K. Weeks, "Lower-limb kinematics of single-leg squat performance in young adults," Physiother. Canada, vol. 66, no. 3, pp. 228-233, 2014.

[37]M. Q. Liu, F. C. Anderson, M. H. Schwartz, and S. L. Delp, "Muscle contributions to support and progression over a range of walking speeds," J. Biomech., vol. 41, no. 15, pp. 3243-3252, 2008.

[38]M. Błażkiewicz, "Muscle force distribution during forward and backward locomotion," Acta Bioeng. Biomech., vol. 15, no. 3, pp. 3-9, 2013.

[39] J. R. Pope, "Comparison of Scaled vs. Ultrasound Based Musculoskeletal Models on Knee Muscle Moments During Single-Leg Squatting,” 2012.

[40]R. Grasso, Y. P. Ivanenko, M. Zago, M. Molinari, G. Scivoletto, and F. Lacquaniti, "Recovery of forward stepping in spinal cord injured patients does not transfer to untrained backward stepping,” Exp. brain Res., vol. 157, no. 3, pp. 377-382, 2004.

[41] R. W. M. van Deursen, T. W. Flynn, J. L. McCrory, and E. Morag, "Does a single control mechanism exist for both forward and backward walking?," Gait Posture, vol. 7, no. 3, pp. 214-224, 1998.

[42]T. S. Buchanan, D. G. Lloyd, K. Manal, T. F. Besier, and others, "Estimation of muscle forces and joint moments using a forward-inverse dynamics model,” Med. Sci. Sports Exerc., vol. 37, no. 11, p. 1911, 2005.

[43] M. Sartori, D. G. Llyod, and D. Farina, "Neural Data-Driven Musculoskeletal Modeling for Personalized Neurorehabilitation Technologies,” IEEE Trans. Biomed. Eng., vol. 63, no. 5, pp. 879-893, 2016.

[44]M. Sartori et al., "Modeling and Simulating the Neuromuscular Mechanisms regulating Ankle and Knee Joint Stiffness during Human Locomotion,” J. Neurophysiol., vol. 114, no. 4, pp. 2509-2527, 2015.

[45] M. M. van der Krogt, L. Bar-On, T. Kindt, K. Desloovere, and J. Harlaar, "Neuro-musculoskeletal simulation of instrumented contracture and spasticity assessment in children with cerebral palsy,” J. Neuroeng. Rehabil., vol. 13, no. 1, p. 64, 2016.

[46] M. Sartori et al., "Toward modeling locomotion using electromyographyinformed 3D models: application to cerebral palsy,” WIREs Syst. Biol. Med., p. e1368, 2017. 